\title{
Dissecting COPD exacerbations: time to rethink our definition
}

\author{
Bartolome R. Celli
}

Affiliation: Pulmonary and Critical Care Division, Brigham and Women's Hospital, Harvard Medical School, Boston, MA, USA.

Correspondence: Bartolome R. Celli, Pulmonary and Critical Care Division, Brigham and Women's Hospital, Harvard Medical School, 75 Francis Street, Boston, MA 02115, USA. E-mail: bcelliacopdnet.org

@ERSpublications

Variables measured during COPD exacerbations inform about their relationships and exacerbation definition http://ow.ly/3mdc30etU3s

Cite this article as: Celli BR. Dissecting COPD exacerbations: time to rethink our definition. Eur Respir J 2017; 50: 1701432 [https://doi.org/10.1183/13993003.01432-2017].

"Measure what is measurable, and make measurable what is not so."

Galileo Galilei (1564-1642)

The Age of Enlightenment reminded humans of the importance of critical and innovative thinking that had been lost during the middle ages. The cited phrase of Galileo is very appropriate when referring to the field of exacerbations of chronic obstructive pulmonary disease (COPD).

In 1821, almost 200 years ago, René Laennec wrote the following regarding emphysema, or what we today call COPD, "The disease can begin in infancy, and continue for many years. More often cough with mucous catarrh that worsens in winter and in the mornings. Often accompanied by difficult respiration, might end fatally by suffocative catarrh. Once present, dyspnea is constant, worsened by anxiety and exercise and above all by development of acute catarrh" [1]. In addition to the very accurate progression of COPD (citing, for example, that the disease could begin in infancy $[2,3]$ ), this summary included the oldest description I could find of exacerbations, when he introduced the term "acute catarrh" as an event that worsened the already present perception of dyspnoea. In addition, he correctly stated that the process is accompanied by difficult ventilation that can be fatal when the catarrh ends in suffocation (respiratory insufficiency). The recent European Respiratory Society/American Thoracic Society guidelines on the management of COPD exacerbations [4] define exacerbations as "episodes of increasing respiratory symptoms, particularly dyspnoea, cough and sputum production, and increased sputum purulence". It is amazing that in the era of high-throughput technology and personalised medicine, the definition has not only not changed, but rather decreased in its content, not differentiating between the "acute" and the "suffocative" catarrh described by Laennec almost 200 years ago.

It is in this context, that the pilot study by Noell et al. [5] in this issue of the European Respiratory Journal is novel. They not only followed Galileo's axiom, quantifying 69 variables including clinical, routine laboratory, microbiological, cytokines and imaging, but also analysed them over time (within $72 \mathrm{~h}$ of hospitalisation and 3 months after discharge) using system network analysis. This integrative technique helps organise and represent visually the relationship between the variables studied without a preconceived hypothesis that could bias the results. The novel use of network analysis has been informative in describing the relationship between COPD and comorbidities $[6,7]$, and the potential role of a B-cell

Received: July 142017 | Accepted after revision: July 202017

Conflict of interest: Disclosures can be found alongside this article at erj.ersjournals.com

Copyright CERS 2017 
immune response in emphysema [8]. This editorial attempts to place in perspective the two main findings reported in this pilot study.

\section{Interpreting the network analysis}

The first observation is that during the acute phase of hospitalisation (first $72 \mathrm{~h}$ ) for an exacerbation, there is a disruption of the organisational interaction that exists amongst all of the clinical and biological variables grouped in modules, compared to that observed after 3 months of recovery. In an analogy, think of it as the chaos that ensues when an initial stroke of the white cue ball hits the well-organised group of numbered balls arranged as a triangle on a pool table. It takes a while for the system to achieve a new steady state with a discernible organisational pattern. The same could be said of the crash of the stock market in 2008 that generated chaos in the relationship among commodities, stocks, funds and different economic variables. It took significant efforts to bring back the system with a new relationship being established amongst the different financial structures. In the example of the pool table, some balls disappear into the pockets, while those left on the table achieve a new relationship that could not be determined easily while the balls were rolling in different directions. In the financial crisis example, the disruption of several market hubs (London, UK; New York, NY, USA; and Frankfurt, Germany) caused profound alterations in the structural relationship amongst all financial centres of the world, leading to a period of disrupted homeokinesis with important repercussions for everybody's financial health. In the study by Noell et al. [5], a similar phenomenon was observed; for example, the modules expressing lung function, which were well connected to those of cytokine expression of inflammation, were disrupted, losing connectivity amongst themselves (such as the practical analogies given above) and becoming isolated from one another (disrupted homeokinesis). However, there are several limitations to their study that render a full interpretation of the results difficult: 1) only the recovery phase at 3 months was studied and this was labelled as the stable state, when in reality the baseline organisational structure of the modules was never measured because there was no evaluation that preceded the exacerbations; 2) The number of patients was relatively small and limited to those admitted to the wards with very few comorbidities, therefore not allowing for comparison with more severe and less severe exacerbations; and 3) there were no "control" patients with COPD without exacerbations or patients without COPD that could serve as the comparators for the purpose of establishing the "normal" or "stable COPD" networks. However, this report provides a push to conduct prospective studies using these novel integrative analytical techniques to provide insights into the effect of interventions on the pattern of response. For example, if the network, with its modules and their relationship, is identified at baseline, then we may evaluate the differential expression of the changes of the network and the modules that may be induced by the administration of antibiotics or corticosteroids, to mention just the currently used medications. Or, if central hubs were identified, how could they be perturbed to more rapidly return to the stable equilibrium or to prevent future episodes of exacerbations? This field is ripe for research $[9,10]$.

\section{Towards an objective taxonomy of exacerbations}

The second set of findings is more conventional but no less interesting and informative, and should help us revisit the definition of exacerbations. Besides the presence of cough and sputum in different degrees, the authors observed, in agreement with Laennec, that during the acute phase of the event, there was a measurable increase in the perception of dyspnoea. This finding has been constant when it has been evaluated prospectively using a dairy card overtime [11] or using a visual analogue scale at the initial encounter in the emergency ward, as was done by PinTo-Plata et al. [12]. In the latter case, the threshold value for an exacerbation was established as 4 on a scale of $0-10$, which is very similar to the value of 3 measured by Noell et al. [5]. Not discussed in detail, but available in the table 2 of the same study, the respiratory rate was higher during the acute phase of the event, a finding that has been reported by others $[12,13]$. Importantly, several studies have shown a large increase in the serum levels of C-reactive protein (CRP) during the event [14-18] and the levels reached are directly related to the severity of the exacerbations [19]. Although in the current report, the threshold value was that of $3 \mathrm{mg} \cdot \mathrm{L}^{-1}$, most of the studies provide values that are generally $>10 \mathrm{mg} \cdot \mathrm{L}^{-1}$. Adding an elevated serum CRP level to symptoms increases the predictive power for exacerbations with areas under the receiver operating characteristic curve $>0.8$ [15-17], considered as clinically useful to predict outcomes. Even though the study by HuRST et al. [17] showed no relationship between CRP level and exacerbation severity, only three of the 90 patients reported in that study were admitted to the hospital, suggesting that the events were mild in nature. Other studies have shown a significantly higher CRP in patients admitted compared to those not admitted to health facilities [16, 19], supporting a relationship between CRP levels and exacerbation severity. Finally, the presence of a high proportion of neutrophils, as seen in the study by Noell et al. [5] or white blood cell count, as shown in other studies have been useful in identifying exacerbations. 
TABLE 1 Analogous to the definitions used for the different acute events in the course of coronary artery disease (CAD), the different acute events of the course of chronic obstructive pulmonary disease (COPD) can be defined using simple, currently available tools

CAD

COPD

Unstable angina
Worsening chest pain
ECG: no myocardial infarction
No laboratory evidence of injury
Myocardial infarction (heart attack)
Chest pain
Abnormal ECG
Abnormal serum enzymes (troponin)

\section{Cardiogenic shock}

Same syndrome plus shock

\author{
Unstable COPD \\ Worsening dyspnoea, VAS $<4$ \\ No decrease in baseline $\mathrm{O}_{2}$ saturation \\ Respiratory rate $<24$ breaths per min \\ Heart rate $<95$ beats per min \\ CRP $<10 \mathrm{mg} \cdot \mathrm{L}^{-1}$ \\ Exacerbation (chest attack) \\ Dyspnoea, VAS $\geqslant 4$ \\ $\mathrm{O}_{2}$ desaturation $<4 \%$ below that of stable state \\ No pneumonia on radiography \\ $\mathrm{CRP}>10 \mathrm{mg} \cdot \mathrm{L}^{-1}$ \\ WBCs $\geqslant 9000$ cells per $\mathrm{mm}^{3}$ \\ BNP $<400 \mathrm{pg} \cdot \mathrm{mL}^{-1}$ \\ D-dimer negative \\ Respiratory failure \\ Same as above plus hypercapnia \\ $\mathrm{PaCO}_{2}>45 \mathrm{mmHg}$
}

These definitions can be further improved as more specific biomarkers are validated. VAS: visual analogue scale (0-10); CRP: C-reactive protein; WBC: white blood cell; BNP: B-type natriuretic peptide; $\mathrm{aCO}_{2}$ : arterial carbon dioxide tension. Information from [27].

It is a fact that the diagnosis of COPD exacerbations based on symptoms only can be confused with other acute events such as pneumonia, pulmonary embolism (PE) or congestive heart failure [20]. However, a good clinical assessment and judicious use of laboratory tests can help decrease the potential confusion with those entities. The absence of infiltrates on the chest radiograph (as in this study) should rule out pneumonia [21]. A negative D-dimer in a patient with low risk for PE or, better yet, a negative computed tomography angiogram (frequently performed on dyspnoeic patients in the emergency room) rules out a pulmonary embolism [22, 23]. Finally a negative troponin or a value of B-type natriuretic peptide $<400 \mathrm{pg} \cdot \mathrm{mL}^{-1}$ makes heart disease an unlikely cause of symptoms [24-26].

We should learn from the advances provided by the evolution of the natural history of coronary artery disease (CAD) [27, 28]. A parallel approach to exacerbations of COPD, shown in table 1, is currently possible as was suggested in this journal in 2007 [29]. CAD is similar to COPD in that it has a long asymptomatic phase in which preventive therapy is effective. Once detected, the disease may be stable and treated as such. In COPD, at a certain point in time, the disease may become more unstable and symptoms worsen; this clinical syndrome could be named "unstable COPD" akin to the "unstable angina" of decompensated patients with CAD. This should prompt modifications in therapy and more careful follow up. If the decompensation is associated with intense dyspnoea ( $>4$ using a $0-10$ scale), worse cough and sputum, tachypnoea ( $>24$ breaths per min), fever, and elevated white cell count ( $>9000$ cells per $\mathrm{mm}^{3}$ ) and CRP $\left(>10 \mathrm{mg} \cdot \mathrm{L}^{-1}\right)$, without evidence of infiltrates in the chest radiography, and negative laboratory tests supportive of other aetiologies, the syndrome could be defined as "exacerbation", or "chest attack" to make it more user friendly, akin to the myocardial infarction or "heart attack" of CAD. Finally, if the same syndrome is accompanied by hypoxaemia [30] with or without elevation of carbon dioxide tension in the arterial blood, the syndrome is defined as "ventilatory failure", akin to the "cardiogenic shock" in CAD. These definitions have the advantage of being practical and of providing a clear guide to clinicians and for the planning of clinical trials. For the clinician, "unstable COPD", although symptomatic, is associated with a low probability of mortality, as has been shown in several prospective trials [31, 32], and therefore could be treated in the ambulatory setting. For the researcher, the different exacerbation states would allow a better planning of trials directed at the specific severity, decreasing the gunshot approach now used.

It is time we stop defining a COPD exacerbations only by its symptoms. The tools are available to be more precise and following the guidance provided by Galileo $>300$ years ago, to measure what is measurable and make measurable what is not. As supported by NoELl et al. [5] in this pilot work and that of many others before them, we can all push this field forward. 


\section{References}

1 Laennec R. A treatise on the diseases of the chest. London, T. Underwood and C. Underwood, 1821.

2 Lange $\mathrm{P}$, Celli B, Agusti A, et al. Lung-function trajectories leading to chronic obstructive pulmonary disease. $N$ Engl J Med 2015; 373: 111-122.

3 Martinez FD. Early-life origins of chronic obstructive pulmonary disease. N Engl J Med 2016; 375: 871-878.

4 Wedzicha J, Miravitlles M, Hurst JR, et al. Management of COPD exacerbations: a European Respiratory Society/ American Thoracic Society guideline. Eur Respir J 2017; 49: 1600791.

5 Noell G, Cosío BG, Faner R, et al. Multi-level differential network analysis of COPD exacerbations. Eur Respir J 2017; 50: 1700075 .

6 Jensen AB, Moseley PL, Oprea TI, et al. Temporal disease trajectories condensed from population-wide registry data covering 6.2 million patients. Nat Commun 2014; 5: 4022.

7 Divo MJ, Casanova C, Marin JM, et al. COPD comorbidities network. Eur Respir J 2015; 46: 640-650.

8 Faner R, Cruz T, Casserras T, et al. Network analysis of lung transcriptomics reveals a distinct B-cell signature in emphysema. Am J Respir Crit Care Med 2016; 193: 1242-1253.

9 Barabasi AL, Gulbahce N, Loscalzo J. Network medicine: a network-based approach to human disease. Nat Rev Genet 2011; 12: 56-68.

10 Loscalzo J, Barabasi AL. Systems biology and the future of medicine. Wiley Interdiscip Rev Syst Biol Med 2011; 3: 619-627.

11 Patel IS, Seemungal TA, Wilks M, et al. Relationship between bacterial colonisation and the frequency, character, and severity of COPD exacerbations. Thorax 2002; 57: 759-764.

12 Pinto-Plata VM, Livnat G, Girish M, et al. Systemic cytokines, clinical and physiological changes in patients hospitalized for exacerbation of COPD. Chest 2007; 131: 37-43.

13 Franciosi LG, Page CP, Celli BR, et al. Markers of exacerbation severity in chronic obstructive pulmonary disease. Respir Res 2006; 7: 74

14 Perera WR, Hurst JR, Wilkinson TM, et al. Inflammatory changes, recovery and recurrence at COPD exacerbation. Eur Respir J 2007; 29: 527-534.

15 Miravitlles M, Moragas A, Hernandez S, et al. Is it possible to identify exacerbations of mild to moderate COPD that do not require antibiotic treatment? Chest 2013; 144: 1571-1577.

16 Bozinovski S, Hutchinson A, Thompson M, et al. Serum amyloid A is a biomarker of acute exacerbations of chronic obstructive pulmonary disease. Am J Respir Crit Care Med 2008; 177: 269-278.

17 Hurst JR, Donaldson GC, Perera WR, et al. Use of plasma biomarkers at exacerbation of chronic obstructive pulmonary disease. Am J Respir Crit Care Med 2006; 174: 867-874.

18 Dev D, Wallace E, Sankaran R, et al. Value of C-reactive protein measurements in exacerbations of chronic obstructive pulmonary disease. Respir Med 1998; 92: 664-667.

19 Karadeniz G, Polat G, Senol G, et al. C-reactive protein measurements as a marker of the severity of chronic obstructive pulmonary disease exacerbations. Inflammation 2013; 36: 948-953.

20 Vogelmeier CF, Criner GJ, Martinez FJ, et al. Global Strategy for the Diagnosis, Management, and Prevention of Chronic Obstructive Lung Disease 2017 Report: GOLD Executive Summary. Eur Respir J 2017; 49 : 1700214.

21 Mandell LA, Wunderink RG, Anzueto A, et al. Infectious Diseases Society of America/American Thoracic Society consensus guidelines on the management of community-acquired pneumonia in adults. Clin Infect Dis 2007; 44: Suppl 2, S27-S72.

22 van der Hulle T, Cheung WY, Kooij S, et al. Simplified diagnostic management of suspected pulmonary embolism (the YEARS study): a prospective, multicentre, cohort study. Lancet 2017; 390: 289-297.

23 Harringa JB, Bracken RL, Nagle SK, et al. Negative D-dimer testing excludes pulmonary embolism in non-high risk patients in the emergency department. Emerg Radiol 2017; 24: 273-280.

24 Nishimura K, Nishimura T, Onishi K, et al. Changes in plasma levels of B-type natriuretic peptide with acute exacerbations of chronic obstructive pulmonary disease. Int J Chron Obstruct Pulmon Dis 2014; 9: 155-162.

25 Adrish M, Nannaka VB, Cano EJ, et al. Significance of NT-pro-BNP in acute exacerbation of COPD patients without underlying left ventricular dysfunction. Int J Chron Obstruct Pulmon Dis 2017; 12: 1183-1189.

26 Buchan A, Bennett R, Coad A, et al. The role of cardiac biomarkers for predicting left ventricular dysfunction and cardiovascular mortality in acute exacerbations of COPD. Open Heart 2015; 2: e000052.

27 Libby P. Mechanisms of acute coronary syndromes. N Engl J Med 2013; 369: 883-884.

28 Klingenberg R, Aghlmandi S, Raber L, et al. Improved risk stratification of patients with acute coronary syndromes using a combination of hsTnT, NT-proBNP and hsCRP with the GRACE score. Eur Heart J Acute Cardiovasc Care 2016: 2048872616684678.

29 Celli BR, Barnes PJ. Exacerbations of chronic obstructive pulmonary disease. Eur Respir J 2007; 29: 1224-1238.

30 Hurst JR, Donaldson GC, Quint JK, et al. Domiciliary pulse-oximetry at exacerbation of chronic obstructive pulmonary disease: prospective pilot study. BMC Pulm Med 2010; 10: 52.

31 Martinez FJ, Vestbo J, Anderson JA, et al. Effect of fluticasone furoate and vilanterol on exacerbations of COPD in patients with moderate airflow obstruction. Am J Respir Crit Care Med 2017; 195: 881-888.

32 Mullerova H, Maselli DJ, Locantore N, et al. Hospitalized exacerbations of COPD: risk factors and outcomes in the ECLIPSE cohort. Chest 2015; 147: 999-1007. 